\title{
Prognostic value of pretreatment neutrophil-to- lymphocyte and platelet-to-lymphocyte ratios in patients with stage III/IV NK/T-cell lymphoma, nasal type (NTCL)
}

Joohwan Park

Seoul National University Hospital

Miso Kim (D misokim85@gmail.com )

Seoul National University Hospital

Tae-Min Kim

Seoul National University Hospital

Bhumsuk Keam

Seoul National University Hospital

Dong-Wan Kim

Seoul National University Hospital

Yoon Kyung Jeon

Seoul National University Hospital

Dae Seog Heo

Seoul National University Hospital

Research article

Keywords: Extranodal NK/T-cell lymphoma, nasal type; neutrophil-to-lymphocyte ratio; platelet-tolymphocyte ratio; and prognosis

Posted Date: September 23rd, 2019

DOI: https://doi.org/10.21203/rs.2.14783/v1

License: (c) (1) This work is licensed under a Creative Commons Attribution 4.0 International License. Read Full License 


\section{Abstract}

Systemic inflammatory response index such as neutrophil-to-lymphocyte ratio (NLR) or platelet-tolymphocyte ratio (PLR) has been investigated as prognostic indicators in various tumors including diffuse large B-cell and peripheral T-cell lymphomas. The aim of this study is to evaluate the prognostic values of the pretreatment NLR and PLR in patients with advanced, extranodal NK/T-cell lymphoma, nasal type (NTCL). Sixty patients with newly diagnosed, stage III/IV NTCL between January 2000 and August 2015 were evaluated retrospectively. Correlations of pre-NLR and -PLR with other clinical factors were investigated. Kaplan-Meier plot was used to analyze survivals according to pre-NLR and -PLR levels. The prognostic effects of variables were investigated using a Cox proportional regression model. Based on the optimized cut-off levels, patients were classified into two different groups. After a median follow up of 15 months, the median overall survival (OS) in the low and high pre-NLR groups was 33.4 and 6.6 months, respectively. In multivariate analysis, high pre-NLR was significantly associated with reduced OS (hazard ratio [HR] 3.59, 95\% $\mathrm{Cl} 1.77-7.26 ; \mathrm{p}<.001$ ). On the contrary, the median OS in the low and high pre-PLR was 6.2 and 22.3 months, respectively. Multivariate analysis revealed that low pre-PLR was in a significant correlation with the worse OS (HR 3.78, 95\% Cl 1.79-7.95; $p<.001$ ). The high pre-NLR and low pre-PLR are independent poor prognostic factors for reduced survivals of patients with advanced NTCL, and might provide additional prognostic powers to identify high-risk, advanced NTCL patients.

\section{Background}

Extranodal natural killer/T-cell lymphoma, nasal type (NTCL) is a distinct subtype of non-Hodgkin lymphoma, which is characterized by prominent necrosis and cytotoxic phenotype related to the EpsteinBarr virus (EBV) (1). It is more prevalent in Asia, Central, and South America while uncommon in other parts of the world, including the USA and Europe (2). Its incidence rate in Korea is approximately $6.3 \%$ among all non-Hodgkin's lymphoma with a male predominance. An early phase of NTCL seems to be localized to upper aerodigestive tract (UAT), but later disseminates to extranodal sites. NTCL shows an aggressive clinical behavior with distinct clinicopathologic characteristics. Despite radiotherapy and chemotherapy, the prognosis is poor with 5-year survival rate less than $50 \%$ (3). Therefore, ongoing research is needed to identify optimal therapeutic targets and prognostic factors to predict the heterogeneous clinical behaviors of this entity.

Although International Prognostic Index (IPI) has been proven to be useful in non-Hodgkin's lymphomas (4), its prognostic impact has not been widely accepted for NTCL. Based on IPI score, some patients classified as a low risk exhibited a poor prognosis (5). This prompted several studies to discover novel prognostic markers or indices such as the Korean Prognostic Index (KPI) (6), Prognostic Index of Natural Killer lymphoma (PINK) (7), circulating EBV-DNA level, Ki-67 expression, and local tumor invasiveness (8-10).

It has been demonstrated that pro-inflammatory chemokines and cytokines within the tumor microenvironment can lead to the recruitment of inflammatory mediators (11). On the basis of this 
evidence, various markers of inflammation have been studied for their association with cancer progression and prognosis (12). A series of prognostic parameters associated with a systemic inflammatory response include C-reactive protein (CRP), the Glasgow Prognostic Score (GPS) and hematological parameters $(13,14)$, of which, neutrophil-to-lymphocyte ratio (NLR) was closely related to the poor survival in diffuse large B-cell lymphoma (DLBCL) as well as various solid tumors $(15,16)$. Platelets, also part of the inflammatory response, are known to promote the tumorigenesis by interacting with tumor cells and protecting them from natural killer cell-mediated lysis (17). Hence, the platelet-tolymphocyte ratio (PLR) has been studied as a prognostic marker in cancer (18).

Although previous studies have demonstrated the potential prognostic significance of NLR and PLR in a variety of cancers, little is known about their prognostic impact in advanced NTCL. In the present study, we evaluated the value of the pre-treatment NLR and PLR in predicting prognosis in stage III/IV NTCL.

\section{Methods}

\section{Patient selection}

A total of 60 patients with stage III/IV NTCL were newly diagnosed between January 2000 and August 2015 at Seoul National University Hospital. The criteria for case inclusion and exclusion were as follows: (1) Histologically proven NTCL according to the WHO classification. (2) Previously untreated, stage III/IV NTCL. (3) Patients who received chemotherapy for a curative intent. (4) A complete set of clinical information and follow-up data. (5) Patients with any clinical, radiologic or microbiologic evidence of active infection were excluded. (6) Patients on steroids before determination of NLR and PLR were also excluded.

\section{Data collection}

The staging work-up data included complete blood count; blood chemistry including lactate dehydrogenase (LDH); computed tomography (CT) scans of the neck, chest, and abdomen; bone marrow (BM) examination; and otolaryngologic examination of UAT. UAT-NTCL and non-UAT (NUAT)-NTCL were defined as previously described (2). The clinical and pathological factors collected for analysis were as follows: age, sex, B symptoms, Eastern Cooperative Oncology Group performance status (ECOG PS), Ann Arbor stage, number of extranodal sites, IPI score, KPI and PINK. Absolute neutrophil count, absolute lymphocyte count, and absolute platelet count in peripheral blood were derived from the standard automated complete blood counts. NLR and PLR were obtained using the formula NLR = absolute neutrophil count/ absolute lymphocyte count and PLR = absolute platelet count/ absolute lymphocyte count. Pre-treatment (Pre)-NLR and -PLR were measured just before the first cycle of chemotherapy. This study was reviewed and approved by the Institutional Review Board of each participant center and was conducted in accordance with the precepts established by the Declaration of Helsinki (H-1605-060-761). 


\section{Statistical analysis}

Clinical variables were compared between the groups by Pearson chi-square or Fisher exact tests, as appropriate. Overall survival (OS) was measured from the date of diagnosis until either the time of death from any cause or the last follow-up visit. Progression-free survival (PFS) was measured from the date of initial treatment to the date of disease progression, death, or the last follow-up visit. Survival analyses were performed by the Kaplan-Meier method and significant differences between groups were identified using the log-rank test. Univariate and multivariate survival analyses were performed according to a Cox proportional hazards model. Variables with clinical significance and a significance level of $<0.05$ were used for covariate entry. Variables with a $P$ value $>0.10$ were removed during a backward stepwise analysis. NLR and PLR cut-off optimization was performed using the web-based R software package Cutoff Finder (http://molpath.charite.de/cutoff/) (19). All statistical tests were two-sided, with significance defined as $P<0.05$. All analyses were performed using SPSS, version 21.0 (IBM Corporation, Armonk, NY, USA).

\section{Results}

\section{Patient Characteristics}

The baseline characteristics of 60 patients are described in Table 1. Patients in our study group showed the male predominance with a median age of 49 years (range, 18 to 73 years). UAT-NTCL was diagnosed in 32 patients (53.3\%) and NUAT-NTCL in 28 patients (46.7\%). The majority of patients (81.7\%) had elevated LDH levels. The presence of B symptoms was observed in 39 patients (65\%) and 46 patients $(76.7 \%)$ had two or more extranodal sites. Hemophagocytic histiocytes of BM were observed in twelve patients $(20 \%)$. IPI, KPI, and PINK scores also suggested that the majority of patients $(78.3 \%, 88.3 \%$, and $71.7 \%$, respectively) were in the high-risk category. The NLR ranged from 0.1 to 31.9 with a mean value of 3.0 and the PLR ranged from 1.5 to 1126.0 with a median value of 223.4. The best cut-off points for NLR and PLR were chosen to yield the highest difference in OS. The most discriminatory cut-offs for NLR and PLR were 3.2 and 133, respectively (Figure 1). Based on these cutoff values used in our subsequent statistical analyses, we classified the patients into the high and low NLR/PLR groups.

\section{Clinicopathological factors associated with pre-NLR and -PLR}

Potential relationships between pre-NLR/PLR and other clinico-pathological factors were shown in Table 2. At baseline, thirty-four patients (56.7\%) had a low pre-NLR of $<3.2$ and twenty-six patients $(43.3 \%)$ had a high pre-NLR of $\geq 3.2$. Twenty eight patients $(40 \%)$ had a low pre-PLR of $<133$ and forty two patients $(60 \%)$ had a high pre-PLR of $\geq 133$. No significant correlations were detected between pre-NLR and sex, 
age, UAT/NUAT presentation, LDH level, number of extranodal sites, BM involvement, Ann Arbor stage, IPI, $\mathrm{KPI}$ and PINK. However, high pre-NLR was found to be in significant correlation with B symptoms $(p=$ $.005)$ and ECOG PS ( $p=.007)$. As for low pre-PLR, it was significantly correlated with LDH level $(p=.024)$, BM involvement $(p=.017)$, and hemophagocytosis $(p=.031)$, but not with any other factors examined here. Specifically, high pre-NLR was more likely to exhibit B symptoms and was significantly correlated with a poor ECOG PS. On the contrary, low pre-PLR was positively correlated with the presence of BM involvement and elevated LDH level.

\section{Treatment response according to pre-NLR and -PLR}

All patients received first-line chemotherapy consisting of ifosfamide, methotrexate, etoposide, and prednisolone (IMEP) plus L-asparaginase or pegaspargase ( $n=30)$; IMEP $(n=21)$, cyclophosphamide, doxorubicin, vincristine, and prednisolone ( $\mathrm{CHOP}, \mathrm{n}=6)$; bleomycin, vincristine, and prednisolone (BVP, $n=2$ ); cyclophosphamide, vincristine, doxorubicin, bleomycin, procarbazine, and prednisolone (COPBLAM$V, n=1)$. The data on evaluation of treatment response to the initial therapy were available in 56 patients (93.3\%) (Table 3). Complete remission was observed in 25 patients (44.6\%), partial remission in 9 patients (16.1\%), stable disease in 19 patients (33.9\%), and progressive disease in the remaining 3 patients (5.4\%), resulting in the objective response rate (ORR) of $60.7 \%$. However, the objective response rate was not significantly different between two patient groups classified by pre-NLR $(63.6 \%$ vs $56.5 \%, p=$ $.592)$ and pre-PLR (53.3\% vs $63.4 \%, p=.494)$. This indicates that both pre-NLR and -PLR might not serve as a predictive factor for response to chemotherapy in patients with advanced NTCL.

\section{Prediction of survival}

The median follow-up time was 15 months (range, 0.9 - 148.9 months). Forty-two patients (70\%) died as of September 2016. Kaplan-Meier estimates of survival for patients with low or high pre-NLR/PLR levels are shown in Figure 2 and 3, respectively. The Kaplan-Meier curves for the survival revealed that patients with high pre-NLR had significantly worse OS (median OS, 6.6 vs 33.4 months, $p=.017$ ) and PFS (median PFS, 4.2 vs 7.5 months, $p=.031$ ). In contrast, low pre-PLR showed the significantly poor OS (median OS, 22.3 vs 6.2 months, $p=.011$ ) and PFS (median PFS, 6.9 vs 3.0 months, $p=.008$ ).

According to a Cox univariate proportional hazards analysis, the clinical factors associated with reduced OS were high pre-NLR $(\mathrm{HR}=2.06,95 \% \mathrm{Cl} 1.12-3.78 ; p=.020)$, low pre-PLR $(\mathrm{HR}=2.24,95 \% \mathrm{Cl} 1.18-4.26 ; p$ $=.014)$, and ECOG PS $\geq 2(\mathrm{HR}=2.14,95 \% \mathrm{Cl} 1.15-3.98 ; p=.016)$ (Table 4). Multivariate analysis identified high pre-NLR $(\mathrm{HR}=3.59,95 \% \mathrm{Cl} 1.77-7.26 ; p<.001)$, low pre-PLR $(\mathrm{HR}=3.78,95 \% \mathrm{Cl} 1.79-7.95 ; p$ $<.001)$, and number of extranodal sites $\geq 2(\mathrm{HR}=3.39,95 \% \mathrm{Cl} 1.33-8.62 ; p=.011)$ as independent poor prognostic factors for OS. 
Regarding the PFS, the univariate analysis revealed that the clinical factors significantly associated with reduced survival were high pre-NLR $(\mathrm{HR}=1.82,95 \% \mathrm{Cl} 1.05-3.17 ; p=.034)$, low pre-PLR $(\mathrm{HR}=2.16,95 \%$ Cl 1.20-3.90; $p=.010)$, ECOG PS $\geq 2$ (HR = 2.13,95\% Cl 1.21-3.76; $p=.009)$, high PINK score $(\mathrm{HR}=1.98$, $95 \% \mathrm{Cl} 1.03-3.80 ; p=.041)$, and elevated LDH level (HR=2.60,95\% Cl 1.10-6.16; $p=.029)$. However, ECOG PS $\geq 2$ (HR $=2.40,95 \%$ Cl 1.29-4.45; $p=.005)$ was an only independent factor for PFS on a multivariate analysis.

\section{Discussion}

We evaluated the prognostic role of the pre-NLR and -PLR in patients with advanced stage III/IV NTCL. Although both pre-NLR and -PLR did not serve as a predictive factor for the treatment response to various combinations of chemotherapy in this malignancy, they were independent prognostic factors for OS in patients with advanced NTCL, regardless of other prognostic indices. Specifically, high pre-NLR and low pre-PLR were adverse predictors for overall survival in multivariate analysis. However, these were not significantly associated with PFS in multivariate analysis. One of the possible reasons explaining this could be due to the cut-off points chosen in our study to give the highest difference in OS. Indeed, both markers retained the prognostic value for PFS as well when the cut-off points to yield the highest difference in PFS were selected (data not shown).

Although the mechanism on how NLR and PLR might contribute to the tumor microenvironment remains unclear, it is well-known that the inflammation could result in neutrophilia, leukocytosis, thrombocytosis, and lymphocytopenia. Neutrophils within the tumor microenvironment produce a variety of cytokines such as interleukin-6, interleukin-10, and tumor necrosis factor a, which are known to promote the tumor progression (20). Moreover, there are also previous studies on the immunosuppressive function of neutrophils exerted on the cytotoxic activity of immune cells such as lymphocytes and natural killer cells $(21,22)$. On the contrary, lymphocyte is a key component of immune surveillance to exert the antibodydependent cellular cytotoxicity against the tumor cells. In the light of these properties of the inflammation, the inflammatory response particularly within the tumor microenvironment might eventually influence the tumor survival, which could be measurable by the change in NLR and PLR.

What is then the potential source of chronic inflammation contributing to the NK/T-cell lymphoma? One explanation is that the necrosis and inflammation characteristic of NTCL might originate from the Epstein-Barr virus (EBV) infection strongly associated with NTCL. Hence, it will be interesting to investigate the relationship of EBV status with the inflammation markers such as NLR and PLR in terms of the survival outcome.

Many previous studies on the prognostic value of NLR in various lymphomas reported the consistent results with our study. However, the low pre-PLR was significantly associated with the poor prognosis in our study. This is inconsistent with several reports demonstrating the association of the high PLR with the poor survival outcome $(18,23)$. There are several possible explanations for this discrepancy. First, thrombocytopenia itself is the poor prognostic factor in lymphomas as reported by other recent studies 
$(7,24)$. Second, the hemophagocytosis known as the poor prognostic factor, is correlated with low prePLR in this study (25). Thus, further study on a large scale is needed to confirm the prognostic role of PLR in advanced NTCL.

The current study had several limitations. First, this was a single-institutional retrospective study with a small population size. Second, the optimal cut-off points of NLR and PLR have not been established. Although an NLR cut-off of 3 or 5 is commonly used in the prognostic setting of some types of cancer, it is not universally applied. Similarly, cut-off points of PLR used in cancers range from 160 to 300 . We used the cut-off points of 3.2 and 133 for NLR and PLR, respectively. Therefore, more accurate determination of the optimal cut-off points in larger prospective studies might allow the routine use of these inflammation-based markers as prognostic indicators for NTCL.

In conclusion, our study demonstrates that pretreatment NLR and PLR are independent prognostic factors for the OS in patients with advanced stage NTCL. This suggests that changes in NLR and PLR associated with NTCL could be promising markers for prognosis of NTCL.

\section{Declarations}

\section{Acknowledgement}

We thank SA Ha Ph.D. for her kind help in manuscript preparation.

\section{Authors' contributions}

MSK, TMK, BK, DWK, YKJ, DSH contributed data acquisition. JHP and MSK analyzed the data. JHP, MSK and TMK wrote and revised the manuscript. All authors have read and approve the final manuscript.

\section{Funding}

This study was supported by a grant of the Korea Health Technology R\&D Project "Strategic Center of Cell and Bio Therapy for Heart, Diabetes \& Cancer" through the Korea Health Industry Development Institute (KHIDI), funded by the Ministry of Health \& Welfare (MHW), Republic of Korea (grant number: HI17C2085).

\section{Availability of data and materials}

The datasets used and/or analyzed during the current study available from the corresponding author on reasonable request 


\section{Consent for publication}

Not Applicable

\section{Competing Interest}

The authors declare that they have no competing interest

\section{References}

1. Campo E, Swerdlow SH, Harris NL, Pileri S, Stein H, Jaffe ES. The 2008 WHO classification of lymphoid neoplasms and beyond: evolving concepts and practical applications. Blood. 2011;117(19):5019-32.

2. Kim TM, Lee SY, Jeon YK, Ryoo BY, Cho GJ, Hong YS, et al. Clinical heterogeneity of extranodal NK/Tcell lymphoma, nasal type: a national survey of the Korean Cancer Study Group. Annals of oncology : official journal of the European Society for Medical Oncology. 2008;19(8):1477-84.

3. Chim CS, Ma SY, Au WY, Choy C, Lie AK, Liang R, et al. Primary nasal natural killer cell lymphoma: long-term treatment outcome and relationship with the International Prognostic Index. Blood. 2004;103(1):216-21.

4. A predictive model for aggressive non-Hodgkin's lymphoma. The New England journal of medicine. 1993;329(14):987-94.

5. Lee J, Kim WS, Park YH, Park SH, Park KW, Kang JH, et al. Nasal-type NK/T cell lymphoma: clinical features and treatment outcome. British journal of cancer. 2005;92(7):1226-30.

6. Lee J, Suh C, Park YH, Ko YH, Bang SM, Lee JH, et al. Extranodal natural killer T-cell lymphoma, nasal-type: a prognostic model from a retrospective multicenter study. Journal of clinical oncology : official journal of the American Society of Clinical Oncology. 2006;24(4):612-8.

7. Kim SJ, Yoon DH, Jaccard A, Chng WJ, Lim ST, Hong H, et al. A prognostic index for natural killer cell lymphoma after non-anthracycline-based treatment: a multicentre, retrospective analysis. The Lancet Oncology. 2016;17(3):389-400.

8. Hsieh PP, Tung CL, Chan AB, Liao JB, Wang JS, Tseng HH, et al. EBV viral load in tumor tissue is an important prognostic indicator for nasal NK/T-cell lymphoma. American journal of clinical pathology. 2007;128(4):579-84.

9. Jiang L, Li P, Wang H, Liu J, Zhang X, Qiu H, et al. Prognostic significance of Ki-67 antigen expression in extranodal natural killer/T-cell lymphoma, nasal type. Medical oncology (Northwood, London, England). 2014;31(10):218. 
10. Kim TM, Park YH, Lee SY, Kim JH, Kim DW, Im SA, et al. Local tumor invasiveness is more predictive of survival than International Prognostic Index in stage I(E)/II(E) extranodal NK/T-cell lymphoma, nasal type. Blood. 2005;106(12):3785-90.

11. Mantovani A, Allavena P, Sica A, Balkwill F. Cancer-related inflammation. Nature. 2008;454(7203):436-44.

12. Colotta F, Allavena P, Sica A, Garlanda C, Mantovani A. Cancer-related inflammation, the seventh hallmark of cancer: links to genetic instability. Carcinogenesis. 2009;30(7):1073-81.

13. Dolan RD, McSorley ST, Horgan PG, Laird B, McMillan DC. The role of the systemic inflammatory response in predicting outcomes in patients with advanced inoperable cancer: Systematic review and meta-analysis. Critical reviews in oncology/hematology. 2017;116:134-46.

14. Shrotriya S, Walsh D, Bennani-Baiti N, Thomas S, Lorton C. C-Reactive Protein Is an Important Biomarker for Prognosis Tumor Recurrence and Treatment Response in Adult Solid Tumors: A Systematic Review. PloS one. 2015;10(12):e0143080.

15. Wang J, Zhou X, Liu Y, Li Z, Li X. Prognostic significance of neutrophil-to-lymphocyte ratio in diffuse large B-cell lymphoma: A meta-analysis. PloS one. 2017;12(4):e0176008.

16. Templeton AJ, McNamara MG, Seruga B, Vera-Badillo FE, Aneja P, Ocana A, et al. Prognostic role of neutrophil-to-lymphocyte ratio in solid tumors: a systematic review and meta-analysis. Journal of the National Cancer Institute. 2014;106(6):dju124.

17. Nieswandt B, Hafner M, Echtenacher B, Mannel DN. Lysis of tumor cells by natural killer cells in mice is impeded by platelets. Cancer research. 1999;59(6):1295-300.

18. Templeton AJ, Ace O, McNamara MG, Al-Mubarak M, Vera-Badillo FE, Hermanns T, et al. Prognostic role of platelet to lymphocyte ratio in solid tumors: a systematic review and meta-analysis. Cancer epidemiology, biomarkers \& prevention : a publication of the American Association for Cancer Research, cosponsored by the American Society of Preventive Oncology. 2014;23(7):1204-12.

19. Budczies J, Klauschen F, Sinn BV, Gyorffy B, Schmitt WD, Darb-Esfahani S, et al. Cutoff Finder: a comprehensive and straightforward Web application enabling rapid biomarker cutoff optimization. PloS one. 2012;7(12):e51862.

20. Salazar-Onfray F, Lopez MN, Mendoza-Naranjo A. Paradoxical effects of cytokines in tumor immune surveillance and tumor immune escape. Cytokine \& growth factor reviews. 2007;18(1-2):171-82.

21. Petrie HT, Klassen LW, Kay HD. Inhibition of human cytotoxic T lymphocyte activity in vitro by autologous peripheral blood granulocytes. Journal of immunology (Baltimore, Md : 1950). 1985;134(1):230-4.

22. el-Hag A, Clark RA. Immunosuppression by activated human neutrophils. Dependence on the myeloperoxidase system. Journal of immunology (Baltimore, Md : 1950). 1987;139(7):2406-13.

23. Wang KF, Chang BY, Chen XQ, Liu PP, Wuxiao ZJ, Wang ZH, et al. A prognostic model based on pretreatment platelet lymphocyte ratio for stage IE/IIE upper aerodigestive tract extranodal NK/T cell lymphoma, nasal type. Medical oncology (Northwood, London, England). 2014;31(12):318. 
24. Ochi Y, Kazuma Y, Hiramoto N, Ono Y, Yoshioka S, Yonetani N, et al. Utility of a simple prognostic stratification based on platelet counts and serum albumin levels in elderly patients with diffuse large B cell lymphoma. Annals of hematology. 2017;96(1):1-8.

25. Jia J, Song Y, Lin N, Liu W, Ping L, Zheng W, et al. Clinical features and survival of extranodal natural killer/T cell lymphoma with and without hemophagocytic syndrome. Annals of hematology. 2016;95(12):2023-31.

\section{Tables}

Table 1. Patients' characteristics

\begin{tabular}{llc}
\hline Characteristics & & Number of patients (\%) \\
\hline Sex & Male & $42(70.0)$ \\
& Female & $18(30.0)$ \\
\hline Age & $\leq 60$ years & $49(81.7)$ \\
& $>60$ years & $11(18.3)$ \\
\hline Presentation & UAT & $32(53.3)$ \\
& NUAT & $28(46.7)$ \\
\hline B symptoms & No & $21(35.0)$ \\
& Yes & $39(65.0)$ \\
\hline LDH level & Normal & $11(18.3)$ \\
& Elevated & $49(81.7)$ \\
\hline ECOG PS & $0-1$ & $39(65.0)$ \\
& $\geq 2$ & $21(35.0)$ \\
\hline Number of extranodal sites & $0-1$ & $14(23.3)$ \\
& $\geq 2$ & $46(76.7)$ \\
\hline BM involvement & Absence & $41(68.3)$ \\
& Presence & $18(30.0)$ \\
& Unknown & $1(1.7)$ \\
\hline Hemophagocytosis & Absence & $48(80.0)$ \\
& Presence & $12(20.0)$ \\
\hline Ann Arbor stage & III & $2(3.3)$ \\
& IV & $58(96.7)$ \\
\hline IPI score & $0-2$ & $13(21.7)$ \\
& $3-5$ & $47(78.3)$ \\
\hline KPI score & $0-1$ & $7(11.7)$ \\
& $2-4$ & $53(88.3)$ \\
\hline PINK score & $0-1$ & $17(28.3)$ \\
& $2-4$ & $43(71.7)$ \\
\hline
\end{tabular}

LDH, Lactic Acid Dehydrogenase; ECOG PS, Eastern Cooperative Oncology Group performance status; BM, Bone marrow; IPI, The International Prognostic Index; KPI, Kyoto Prognostic Index; PINK, Prognostic Index of Natural Killer lymphoma 
Table 2. Clinical comparisons between patients with pre-NLR and -PLR

\begin{tabular}{|c|c|c|c|c|c|c|c|}
\hline aracteristics & & $\begin{array}{c}\mathrm{NLR}<3.2 \\
N(\%) \\
(\mathrm{n}=34)\end{array}$ & $\begin{array}{c}N L R \geq 3.2 \\
N(\%) \\
(n=26)\end{array}$ & $p$ & $\begin{array}{c}\text { PLR<133 } \\
\qquad \begin{array}{l}N(\%) \\
(\mathrm{n}=18)\end{array}\end{array}$ & $\begin{array}{c}\text { PLR } \geq 133 N \\
(\%) \\
(n=42)\end{array}$ & $p$ \\
\hline \multirow[t]{2}{*}{$\bar{r}$} & Male & $22(64.7)$ & $20(76.9)$ & 0.306 & $14(77.8)$ & $28(66.7)$ & 0.542 \\
\hline & Female & $12(35.3)$ & $6(23.1)$ & & $4(22.2)$ & $14(33.3)$ & \\
\hline \multirow[t]{2}{*}{3} & $\begin{array}{l}\leq 60 \\
\text { years }\end{array}$ & $26(76.5)$ & $23(88.5)$ & 0.320 & $14(77.8)$ & $35(83.3)$ & 0.719 \\
\hline & $\begin{array}{l}>60 \\
\text { years }\end{array}$ & $8(23.5)$ & $3(11.5)$ & & $4(22.2)$ & 7 (16.7) & \\
\hline \multirow[t]{2}{*}{ sentation } & UAT & $20(58.8)$ & $12(46.2)$ & 0.330 & $8(44.4)$ & $24(57.1)$ & 0.366 \\
\hline & NUAT & $14(41.2)$ & $14(53.8)$ & & $10(55.6)$ & $18(42.9)$ & \\
\hline \multirow[t]{2}{*}{ ymptoms } & No & $17(50.0)$ & $4(15.4)$ & 0.005 & $5(27.8)$ & $16(38.1)$ & 0.443 \\
\hline & Yes & $17(50.0)$ & $22(84.6)$ & & $13(72.2)$ & $26(61.9)$ & \\
\hline \multirow[t]{2}{*}{ H level } & Normal & 8 (23.5) & $3(11.5)$ & 0.320 & $0(0)$ & $11(26.2)$ & 0.024 \\
\hline & Elevated & $26(76.5)$ & $23(88.5)$ & & $18(100)$ & $31(73.8)$ & \\
\hline \multirow[t]{2}{*}{ OG PS } & $0-1$ & $27(79.4)$ & $12(46.2)$ & 0.007 & $10(55.6)$ & $29(69.0)$ & 0.315 \\
\hline & $\geq 2$ & $7(20.6)$ & $14(53.8)$ & & $8(44.4)$ & $13(31.0)$ & \\
\hline mber & $0-1$ & $8(23.5)$ & $6(23.1)$ & 0.967 & $5(27.8)$ & $9(21.4)$ & 0.594 \\
\hline əxtranodal sites & $\geq 2$ & $26(76.5)$ & $20(76.9)$ & & $13(72.2)$ & $33(78.6)$ & \\
\hline \multirow[t]{3}{*}{ [ involvement } & Absence & $21(61.8)$ & $20(23.1)$ & 0.271 & $8(44.4)$ & 33 (78.6) & 0.017 \\
\hline & Presence & $12(35.3)$ & $6(76.9)$ & & $9(50.0)$ & $9(21.4)$ & \\
\hline & Unknown & $1(2.9)$ & $0(0)$ & & $1(5.6)$ & $0(0)$ & \\
\hline \multirow[t]{2}{*}{ mophagocytosis } & Absence & $27(79.4)$ & $21(80.8)$ & 0.896 & $11(61.6)$ & $37(88.1)$ & 0.031 \\
\hline & Presence & $7(20.6)$ & $5(19.2)$ & & $7(38.9)$ & $5(11.9)$ & \\
\hline \multirow[t]{2}{*}{ Arbor stage } & III & $2(5.9)$ & $0(0)$ & 0.501 & $2(11.1)$ & $0(0)$ & 0.086 \\
\hline & IV & $32(94.1)$ & $26(100)$ & & $16(88.9)$ & $42(100)$ & \\
\hline \multirow[t]{2}{*}{ score } & $0-2$ & $10(29.4)$ & $3(11.5)$ & 0.122 & $2(11.1)$ & $11(26.2)$ & 0.308 \\
\hline & $3-5$ & $34(70.6)$ & $23(88.5)$ & & $16(88.9)$ & $31(73.8)$ & \\
\hline \multirow[t]{2}{*}{ I score } & $0-1$ & $6(17.6)$ & $1(3.8)$ & 0.126 & $0(0)$ & 7 (16.7) & 0.091 \\
\hline & $2-4$ & $28(82.4)$ & $25(96.2)$ & & $18(100)$ & $35(83.3)$ & \\
\hline \multirow[t]{2}{*}{ JK score } & $0-1$ & $12(35.3)$ & $5(19.2)$ & 0.171 & $3(16.7)$ & $14(33.3)$ & 0.189 \\
\hline & $2-4$ & $22(64.7)$ & $21(80.8)$ & & $15(83.3)$ & $28(66.7)$ & \\
\hline
\end{tabular}

NLR, neutrophil-to-lymphocyte ratio; PLR, platelet-to-lymphocyte ratio; UAT, upper aerodigestive tract; NUAT, non- upper aerodigestive tract; LDH, Lactic Acid Dehydrogenase; ECOG PS, Eastern Cooperative Oncology Group performance status; BM, Bone marrow; IPI, The International Prognostic Index; KPI, Kyoto Prognostic Index; PINK, Prognostic Index of Natural Killer lymphoma

Table 3. Treatment responses according to pre-NLR and -PLR 
\begin{tabular}{llllllll}
\hline Response & Total & NLR $<3.2$ & NLR $\geq 3.2$ & $p$ & PLR $<133$ & PLR $\geq 133$ & $p$
\end{tabular}

\begin{tabular}{ccccc}
$N(\%)$ & $N(\%)$ & $N(\%)$ & $N(\%)$ & $N(\%)$ \\
$(\mathrm{n}=56)$ & $(\mathrm{n}=34)$ & $(\mathrm{n}=26)$ & $(\mathrm{n}=18)$ & $(\mathrm{n}=42)$ \\
\hline
\end{tabular}

\begin{tabular}{llllllll}
\hline CR + PR $34(60.7 \%)$ & $21(63.6 \%)$ & $13(56.5 \%)$ & 0.592 & $8(53.3 \%)$ & $26(63.4 \%)$ & 0.494 \\
\hline
\end{tabular}

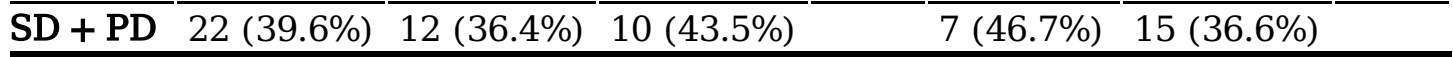

NLR, neutrophil-to-lymphocyte ratio; PLR, platelet-to-lymphocyte ratio; CR, complete remission; PR, partial remission; $\mathrm{SD}$, stable disease; $\mathrm{PD}$, progressive disease

\section{Figures}

(a)

Significant $(p<0.05)$ tests: 10 out of $41(24.4 \%)$

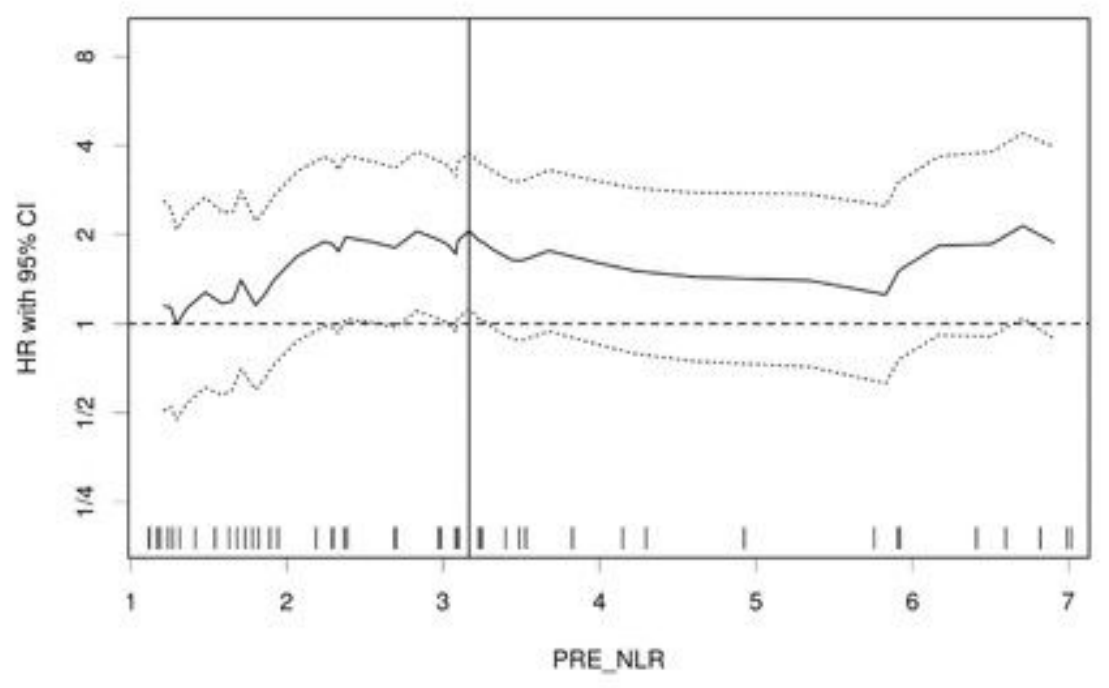

(b) Significant $(p<0.05)$ tests: 7 out of $41(17.1 \%)$

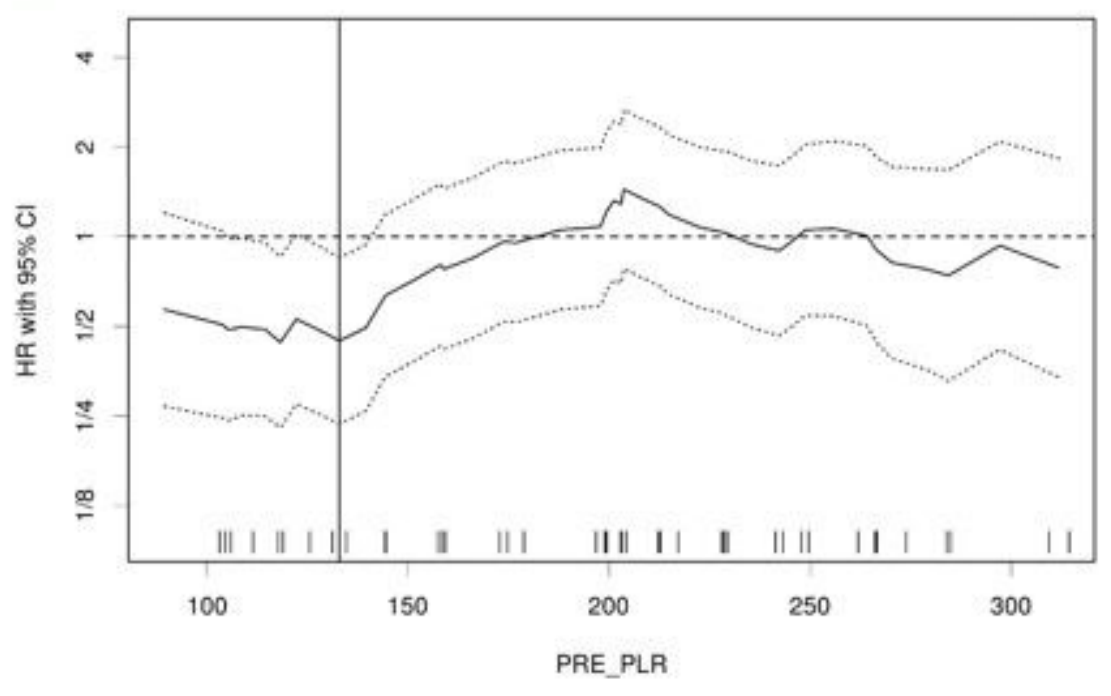

Figure 1 
Hazard ratio (HR) for overall survival in dependence of cutoff points for (a) NLR and (b) PLR. The vertical line designates the optimal cutoff point; NLR, neutrophil-to-lymphocyte ratio; PLR, platelet-to-lymphocyte ratio

(a)

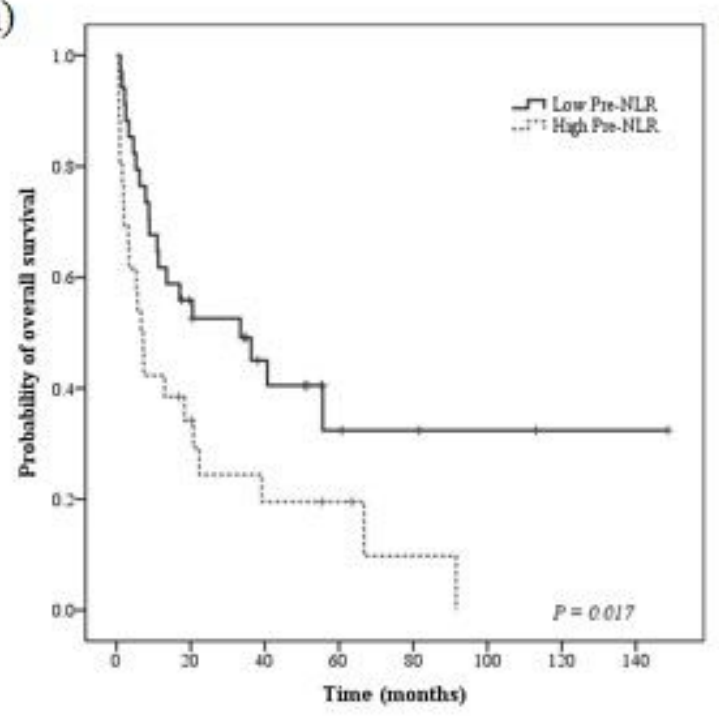

(b)

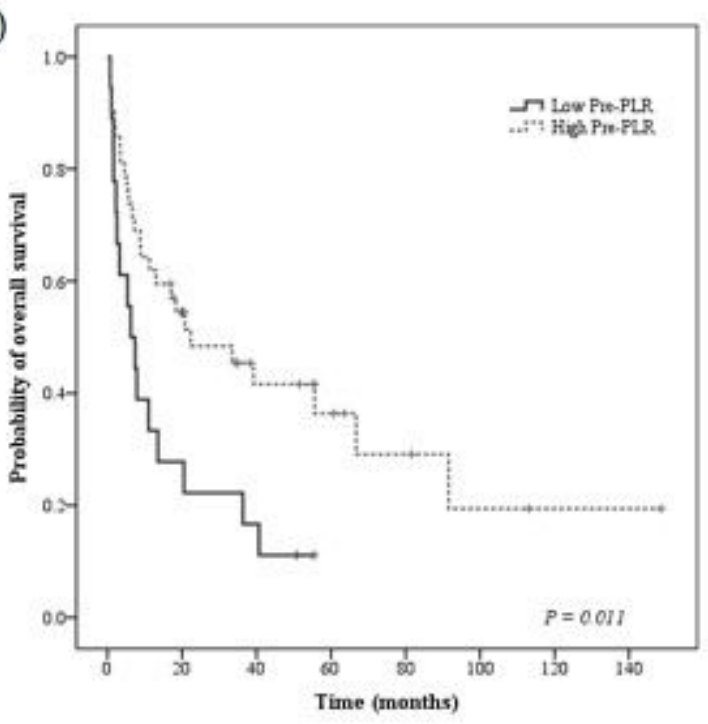

\section{Figure 2}

Kaplan-Meier plots of OS according to pre-treatment NLR (a) and PLR (b): high pre-treatment NLR and low pretreatment PLR are associated with reduced OS; OS, overall survival; NLR, neutrophil-to-lymphocyte ratio; PLR, platelet-to-lymphocyte ratio
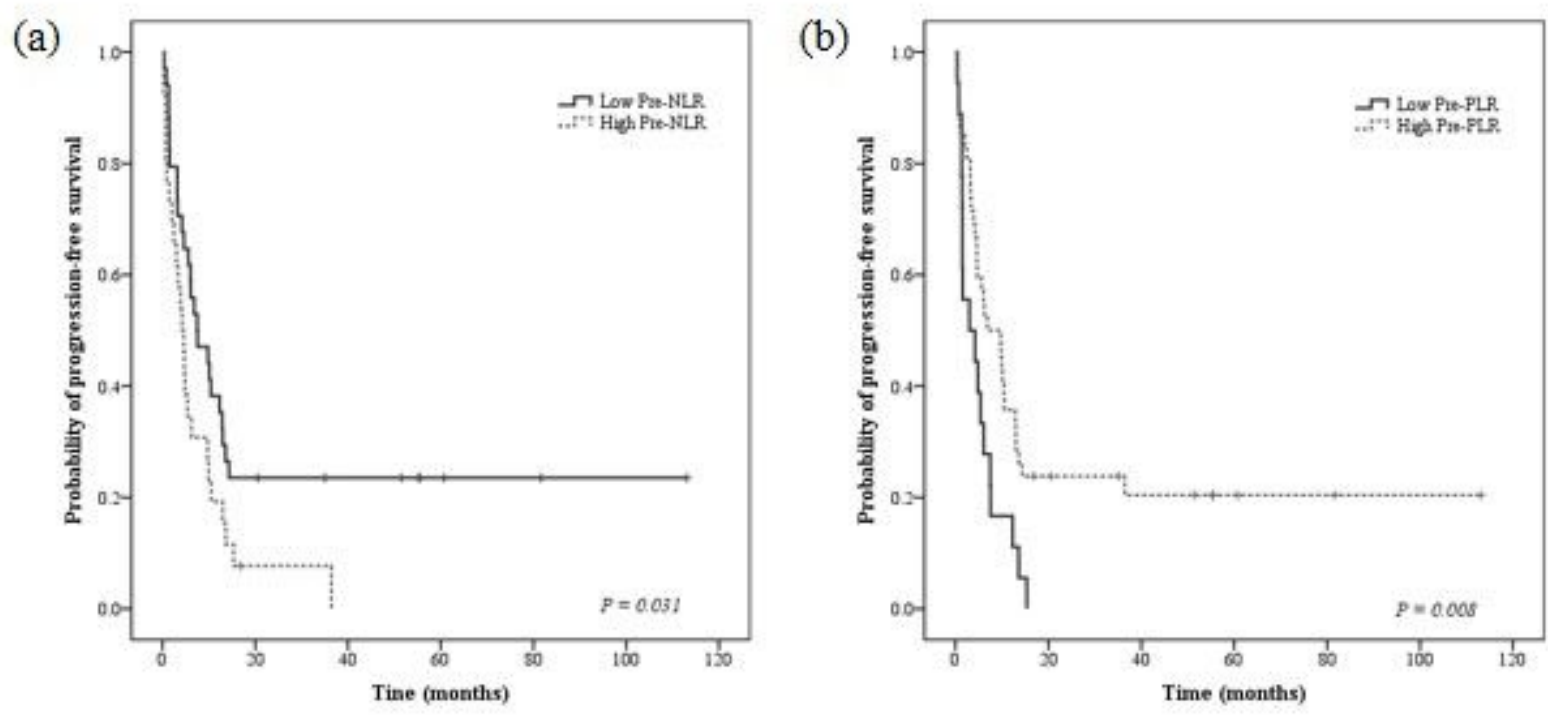

\section{Figure 3}

Kaplan-Meier plots of PFS according to pre-treatment NLR (a) and PLR (b): high pre-treatment NLR and low pretreatment PLR are associated with reduced PFS; PFS, progression-free survival; NLR, neutrophil-tolymphocyte ratio; PLR, platelet-to-lymphocyte ratio 